\title{
Notes sur les Siphonaptères parasites de Carnivores en France
}

\author{
par J.-C. BEAUCOURNU \\ Laboratoire de Parasitologie ( $\mathrm{P}^{r}$ J.-M. DoBY), U.E.R. «Santé dans la Collectivité » \\ Faculté de Médecine, F 35000 Rennes
}

\begin{abstract}
Résumé
Ayant pu étudier, en France, plus de 2.000 siphonaptères parasites de carnivores, ceux-ci appartenant à huit espèces selvatiques, l'auteur fait le point pour ce pays, de nos connaissances sur la biologie, la chorologie et le rôle épidémiologique des principales puces rencontrées: Pulex irritans, Ctenocephalides felis felis, C. canis, Chaetopsylla globiceps, C. homoea, C. matina, C. rothschildi, C. trichosa, Ceratophyllus I. lunatus, Paraceras m. melis. 23 autres espèces sont également signalées dont la rare Amphipsylla sibirica, capturée pour la seconde fois seulement en France.
\end{abstract}

\section{Summary}

After studying more than 2.000 Siphonaptera collected from 8 selvatic species of carnivora in France, the author gives an accurate statement of data on biology, chorology and epidemiological importance, in this country of the collected chief fleas: Pulex irritans, Ctenocephalides felis felis, C. canis, Chaetopsylla globiceps, C. homoea, C. matina, C. rothschildi, C. trichosa, Ceratophyllus l. lunatus, Paraceras m. melis. 23 other species are also signaled, among which the rare Amphipsylla sibirica, collected in France only for the second time. 
L'importance des carnivores est enfin à peu près admise par tous : régulateurs de biocénoses et, à ce titre, à protéger sans réserve mais aussi, malheureusement, réservoirs de virus et de parasites et donc à surveiller étroitement. Sans revenir sur la rage dont le renard (Vulpes vulpes L.) est, semble-t-il, le principal élément de propagation (à l'exclusion des autres carnivores sauvages), rappelons, par exemple, que Rickettsia conori, Leishmania donovani, Trichinella spiralis, Echinococcus multilocularis sont connus, en France, comme agents de parasitoses graves, sinon fatales, chez l'homme : les carnivores en sont le réservoir animal.

En ce qui concerne les puces, renard et blaireau (Meles meles L.) sont classiquement associés comme réservoirs selvatiques d'un parasite mineur et d'intérêt surtout historique, la puce «de l'homme»: Pulex irritans L. est, en effet, une puce de carnivore. Ce parasite, encore sporadiquement banal, était, entre autres, le vecteur de la peste bubonique humaine tant en Afrique du Nord qu'en Europe. Dans nos régions, par conséquent, les réservoirs naturels de l'insecte vecteur, mais non du germe, sont des carnivores et non des rongeurs comme en Asie, ou en Afrique tropicale. Il nous a semblé intéressant de voir, en France, l'importance de cette puce par rapport aux autres espèces peu ou non anthropophiles trouvées sur les carnivores, étant donné son rôle potentiel ou admis dans diverses anthropozoonoses.

Nous étudierons, en outre, les quelques éléments réunis sur les autres siphonaptères de carnivores en France qui nous montreront les interférences possibles entre hôtes sauvages, hôtes domestiques et homme. Nous citerons également les parasites accidentels car ils peuvent apporter quelques données sur le régime alimentaire ou le biotope électif de l'hôte prédateur, donc sur son écologie.

Les parasites de chiens et chats domestiques seront brièvement cités en fonction de nos propres relevés. Les diverses données sur les puces de carnivores sauvages s'appuient sur nos récoltes personnelles (depuis 1956) et les résultats d'une enquête menée auprès de quelques gardes-chasses au cours de l'hiver et du printemps 19711972 (1).

Si l'on excepte le cas des belettes et éventuellement des hermines capturées dans la plupart des régions françaises au cours de nos prospections systématiques, nous n'avions pratiquement, avant cette enquête, que des renseignements fragmentaires sur l'Ouest (Morbihan, Côtes-du-Nord, Ille-et-Vilaine, Mayenne, Sarthe, Maine-et-Loire) ainsi que sur l'Isère (Beaucournu, 1968 ; Beaucournu et Gilot, 1971). Les départements qui ont enrichi nos données grâce à l'aide des gardes-chasses sont les suivants: Alpes de Haute-Provence, Hautes-Alpes, Dordogne, Eure, Haute-Garonne, Lot-et-Garonne, Nièvre, Pyrénées-Atlantiques, Pyrénées-Orientales, Puy-de-Dôme, Tarn, Tarn-et-Garonne, Yonne. L'ensemble de ces départements constitue donc la carte de ce que nous avons réuni, bibliographie mise à part, sur les puces de carnivores.

(1) Nous remercions très vivement MM. Ardoin, Arnaud, Belory, Berjouan, Bertauld, Bonnemaison, Chiapello, Garcia, Keck, Martin A., Martin J., Michez, Pascal, Raso, Roussille, Sauze, Schaeffer, Silvestre, Théron ainsi que les Présidents des Fédérations de chasse correspondantes et tout particulièrement M. J. Lemaire (Nantes) et le $\mathbf{D}^{r} \mathbf{M}$. Reydellet (Gap), membres du Conseil international de la Chasse, pour leur cordiale assistance. 
Les hôtes sauvages suivants ont été étudiés :

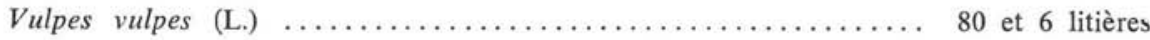

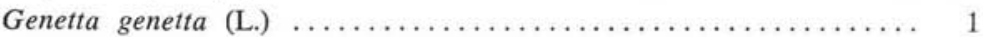

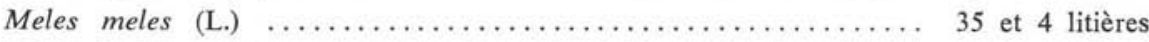

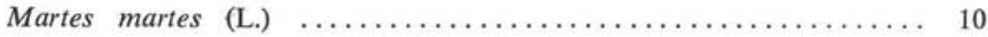

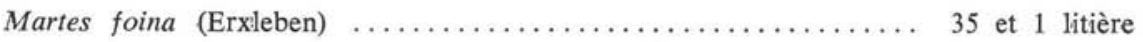

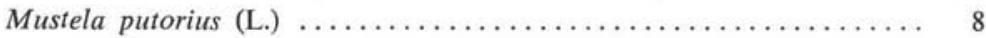

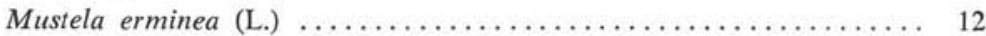

Mustela nivalis nivalis $\mathrm{L}$. et $M$. n. boccamela Bechstein ........ 52 et 1 litière.

33 espèzes de Siphonaptères ont été récoltées sur ces hôtes. La majeure partie (22) sont des déserteurs acquis au contact des proies; les onze autres sont spécifiques d'une ou plusieurs espèces de carnivores ou, du moins, sont régulièrement récoltés sur ces animaux : c'est le cas de Monopsyllus sciurorum.

\section{I. - LES PARASITES.}

\section{Siphonaptères spécifiques de Carnivores (Tableau I)}

\section{Pulicidae}

Pulex irritans L., 1758

En tant que puce selvatique, Pulex irritans est essentiellement inféodé au renard et au blaireau. L'un, comme l'autre, sont parasités un peu plus d'une fois sur deux, mais le nombre moyen de puces semble plus élevé chez le renard que chez le blaireau. En dehors de ces hôtes principaux, nous n'avans noté que la fouine : 5 sur 35 hébergeaient des exemplaires de Pulex irritans. Etant donné l'écologie de ce carnivore, on peut penser qu'il s'est contaminé dans un biotope karstique aux dépens du blaireau.

En effet, à l'état libre, c'est dans les cavités calcaires, naturelles ou non, qu'on récoltera le plus facilement Pulex irritans. Nous l'y avons trouvé en été comme en hiver. Les individus observés étaient rarement dans de vieilles litières, le plus souvent sur le sol ou sur une petite pierre, la proximité de fèces de blaireau ou de renard étant fréquente. Quelques-unes ont été capturées sur les parois entre 0 et $30 \mathrm{~cm}$ de hauteur.

Pulex irritans peut abonder été comme hiver sur des hôtes sauvages et être l'espèce dominante ou seule rencontrée. Toutefois, en altitude, elle se raréfie vite. En France, Smit (1960) en note un exemplaire sur Homo vers 1450-1500 m; Beaucournu et Rault (1962) en signalent un, vers $1800 \mathrm{~m}$, également sur homme, mais capturé dans un éboulis à haute densité en renards, il s'agit manifestement d'un déserteur. Sur hôte sauvage, nous n'en avons qu'un exemplaire, récolté entre 1300 et $1800 \mathrm{~m}$, sur renard, en janvier. Tous les autres, été comme hiver, viennent actuellement d'une altitude inférieure à $800 \mathrm{~m}$. En plaine en tout cas, elle se maintient sous forme imaginale toute l'année avec une densité notable: nous ne connaissons pas ses variations de fréquence sur ses hôtes naturels car nos prélèvements ne sont pas suffisamment étalés. 
Tableau I.

Puces de CARnivores (données personnelles)

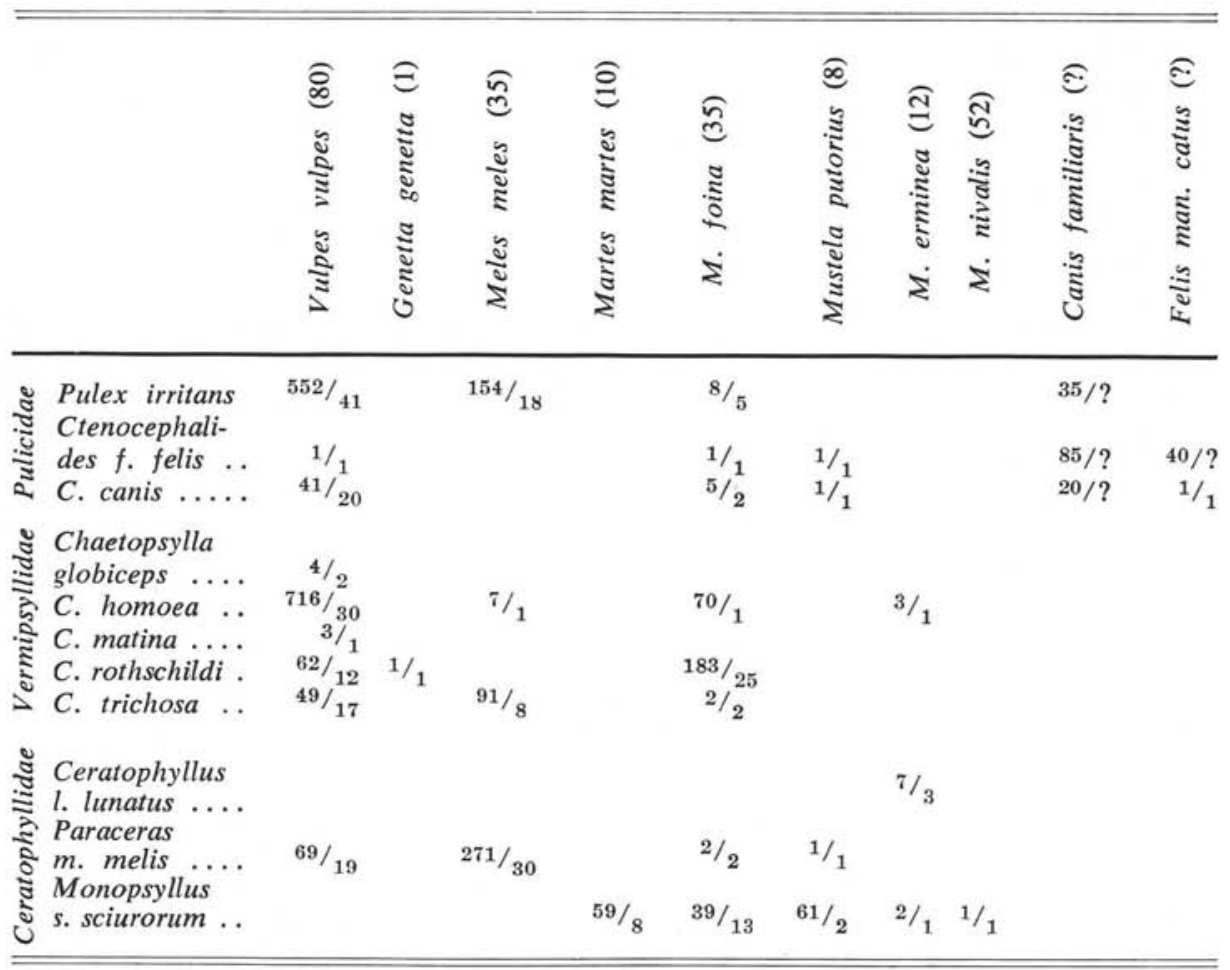

N. B. : Les puces récoltées dans les litières ne sont pas intégrées dans ce tableau Le chiffre supérieur indique le nombre de parasites récoltés, l'inférieur le nombre d'hôtes parasités.

En dehors de ses hôtes selvatiques, Pulex irritans est évidemment bien connue sur l'homme. Toutefois, les conditions microclimatiques des habitations humaines actuelles (faible hygrométrie, plancher compact, produits d'entretien toxiques, aspirateurs) font que Pulex irritans ne peut s'y maintenir. Ce n'est qu'en milieu rural, ou en ville dans des habitations anciennes (plancher disjoint, chauffage déficient), qu'elle se rencontre encore.

Nous avons noté, mois par mois, le nombre de fois (78) où un homme fut trouvé parasité de 1956 à 1972. Nous ne faisons pas état du nombre total de specimens par mois, la surabondance des parasites sur tel individu en mauvaise condition physiologique ou sociale, faussant l'ensemble de nos chiffres. Nos résultats sont réguliers en dehors d'une baisse notable et inexpliquée de mai à juillet inclus, faisant suite à une discrète augmentation en avril-mars (fig. 1). Comme on devait s'y attendre, la baisse hivernale constatée chez de nombreuses puces parasitant les hôtes sauvages n'est pas ressentie ici.

Nous n'avons pas noté de parasitisme du chat par $P$. irritans. Le chien, en revanche, est certainement un excellent hôte. Un ou deux décimètres cubes de litière d'un chien, 
citadin mais vivant dans un niche, nous ont donné, en un mois, plus de 5000 imagos de cette puce, à l'exclusion de toute autre espèce: l'élevage, encore florissant, fut alors arrêté. Nos relevés concernant de chien sont encore très fragmentaires. Il nous paraît cependant que $P$. irritans serait plus fréquente sur cet hôte dans les pays méridionaux.

Le sex-ratio de cette espèce montre toujours une dominance des femelles, mais varie dans nos relevés, d'un hôte à l'autre. Il est de 0,35 (sur 149 exemplaires) sur le blaireau, de 0,46 (sur 100) sur l'homme et de 0,68 (sur 548) sur le renard.

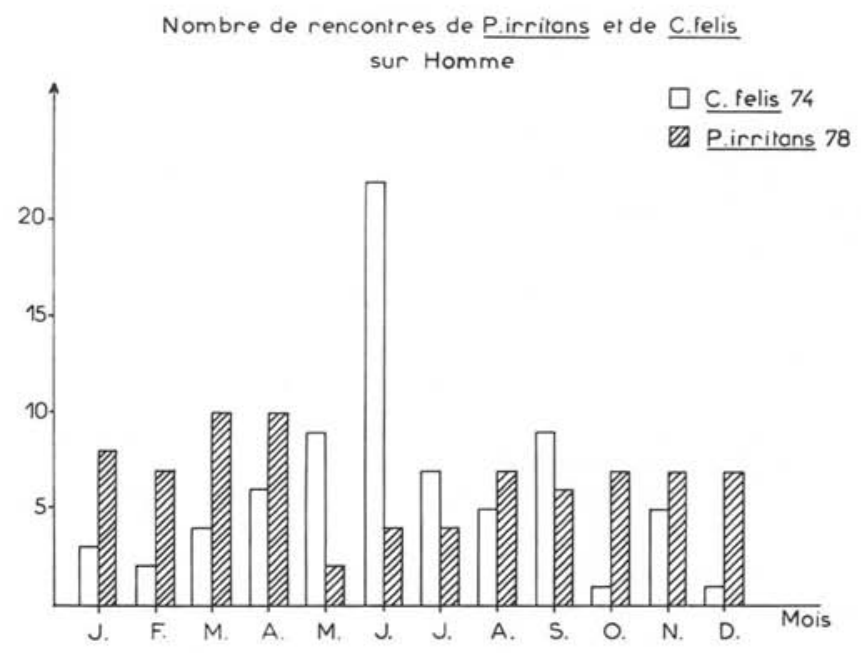

Fig. 1

Nous avons noté dans nos récoltes de nombreux specimens hébergeant, en tant qu'hôtes intermédiaires, des helminthes. caninum.

— une femelle, prélevée sur chien, était porteuse d'un cysticercoïde de Dipylidium

- une trentaine d'exemplaires prélevés sur $V$. vulpes, provenant de deux départements du Sud-Ouest (Lot-et-Garonne et Haute-Garonne), contenaient de nombreuses larves infectantes de Dipetalonema reconditum (2) (Bain et Beaucournu, sous presse). Cette filaire, pour autant que la morphologie des larves III, permette une diagnose spécifique, est nouvelle pour la France et le renard constitue un hôte nouveau. Pulex irritans, excellent hôte intermédiaire et parasite commun au renard, au chien et à l'homme, ne pourrait-il assurer à la fois la persistance du foyer selvatique et la contamination humaine, dans certains cas de filarioses dermiques ou conjonctivales du bassin méditerranéen ? Bien que, en général, soit incriminée une Dirofilaria, il n'est pas impossible que $D$. reconditum en soit quelquefois l'agent.

Nous pensons d'ailleurs que cette communauté d'ectoparasites entre l'homme et divers carnivores est peut-être, accessoirement sans doute, la cause d'autres infections humaines.

(2) Il faut souligner que parmi les puces parasitant le renard, seule P. irritans, dominante à vrai dire dans la région où fut trouvée Dipetalonema, fut trouvée infectée. 
Nous pensons en particulier au Kala-Azar. Rioux et coll. (1969) ont remarquablement montré l'incidence du chien et du renard sur les cas humains. Il est bien évident que le seul hôte intermédiaire est le phlébotome, mais si l'on admet la possibilité de contamination directe chien-homme, il paraît difficile de nier a priori la possibilité de contage mécanique assuré par la puce lorsqu'on sait l'intimité qui peut régner entre le chien familier et l'enfant, par exemple. Il pourrait également en être de même dans la transmission de la fièvre boutonneuse méditerranéenne.

\section{Ctenocephalides felis felis (Bouché, 1835)}

Cette puce, à vaste répartition mondiale pour la forme nominale, celle-ci suivant essentiellement les zones de civilisation moderne hors de la zone paléarctique, est banale en régions d'habitations humaines (urbaines ou rurales) où elle peut dépasser en nombre Pulex irritans et même la supplanter.

Nous n'avons peut-être pas examiné les hôtes selvatiques naturels de cette puce, le chat sauvage, Felis silvestris, par exemple: toujours est-il qu'en dépit de son ubiquité, de son abondance et de son manque de spécificité, $C$. $f$. felis est rare sur des animaux non synanthropes. Nous n'en avons que 4 exemplaires provenant respectivement d'un renard, d'une fouine, d'une litière de cette même espèce et d'un putois.

Par contre, les animaux synanthropes et l'homme sont fréquemment parasités. Il semble que le chat domestique, Felis maniculata catus, soit son hôte principal. Le chien est également très souvent parasité et tout particulièrement le chien de « salon » vivant au contact étroit du chat.

L'homme vient en troisième position comme hôte, accidentel cette fois, de C. f. fells. Nous avons, dans les mêmes conditions que pour la puce «de l'homme», noté mois par mois le nombre de fois (74) où un homme fut trouvé parasité. La courbe est ici plus irrégulière que pour la précédente espèce, mais montre la présence de cette puce durant tout le cyole annuel avec un pic en juin (fig. 1), pic lié peut-être à un optimum climatique. Nos recherches sur le chat domestique sont trop irrégulières pour que nous puissions en faire état. Il est vraisemblable que les variations de fréquence sur l'homme ne sont que le reflet de celles que nous aunions pu observer sur cet hôte normal.

Sur les animaux synanthropes autres que ceux déjà cités, c'est la moins rare des accidentelles et en particulier elle se rencontre sur le rat, Rattus norvegicus, particulièrement semble-t-il dans les zones urbanisées de la sous-région méditerranéenne (3). Signalons enfin que c'est la puce classique des carnivores, et même quelquefois des ongulés, en captivité.

Sous notre climat, en résumé, $C$. $f$. felis se comporte en puce synanthrope inféodée au chat domestique, parasitant secondairement le chien et l'homme. Elle est rare sur les animaux sauvages.

\section{Ctenocephalides canis (Curtis, 1826)}

C. canis est de répartition et de spécificité beaucoup plus étroite que la précédente. Il s'agit manifestement d'un parasite de canidé, le chien, le renard et sans doute le loup, autrefois.

(3) Autre animal synanthrope que nous avons trouvé parasité, mais d'intérêt plus anecdotique que réel, le Martinet noir, Apus apus. 
Pour ce qui concerne le chien, il faut relever que, statistiquement, les individus parasités par $C$. canis seront écologiquement différents de ceux porteurs de $C$. felis. $C$. canis parasitera les chiens vivant en chenil, les chiens de meute, les chiens de berger. C. felis sera surtout, nous l'avons dit, une puce de chien «de salon».

Le chat est exceptionnellement parasité par $C$. canis. Nous n'en avons qu'un exemple et encore s'agissait-il d'un animal qui fréquentait un chenil très infesté, temporairement abandonné de ses hôtes. De même, l'homme semble, d'après nos observations, peu parasité: nous n'en avons que 5 cas (groupant 5 exemplaires) contre 74 (groupant plusieurs centaines de puces) pour $C$. f. felis.

Trois carnivores sauvages figurent dans nos relevés: renard, putois et fouine. Ces deux derniers sont manifestement des hôtes accidentels: un putois a été trouvé porteur d'une seule puce; 2 fouines regroupaient 5 exemplaires.

Avec le renard nous retrouvons un hôte régulier: nous avons une vingtaine d'animaux porteurs, mais la densité par hôte semble beaucoup plus basse que pour Pulex irritans: approximativement on ne trouve que 2 C. canis par hôte contre plus d'une douzaine, en moyenne, de $P$. irritans.

Nous avons récolté cette puce sur le renard tout au long de l'année. En janvier et février, elle se rencontre encore entre 1300 et $2000 \mathrm{~m}$ (Hautes-Alpes, Alpes de HauteProvence, Pyrénées-Orientales). Ce sont alors des individus souvent âgés, jamais néonates, ni ovigères.

C. canis, écologiquement, se montre donc bien différente, et apparemment mieux adaptée aux conditions écologiques de notre pays que $C$. felis. C'est une puce apparaissant comme autochtone, selvatique, ne devenant synanthrope qu'à la suite de l'un de ses deux hôtes naturels, le chien.

Pour $C$. canis, comme pour $C$. felis, nous ne donnons pas de sex-ratio, nos exemplaires récoltés sur hôtes sauvages n'étant pas assez nombreux.

\section{Vermipsyllidae}

Tous les Vermipsyllidae d'Europe appartiennent au genre Chaetopsylla Kohaut, 1903. Ce sont des puces de carnivores (4), écologiquement des puces de fourrure (5) et dont les imagos ne se rencontrent que pendant la saison froide.

Comme il est de règle pour d'autres puces hivernales, la période d'apparition des adultes sera d'autant plus étalée, à latitude égale, que l'altitude sera plus élevée, dans les limites écologiques de l'hôte évidemment.

Nos captures concernent 5 espèces: Chaetopsylla globiceps, C. homoea, C. matina, C. rothschildi et $C$. trichosa.

Chaetopsylla tuberculaticeps (Bezzi, 1890) parasite de l'ours brun Ursus arctos L., signalée de France, n'a pas été revue par nous, faute évidemment d'avoir pu examiner Népal.

(4) A l'exception de C. gracilis Lewis 1971, inféodée à un lagomorphe du genre Ochotona, au

(5) Ou puces d'hôtes: ne se rencontrent à l'état imaginal que, pratiquement, sur l'animal parasité ; elles s'opposent aux puces de nid ou de litière qui ne vont sur l'hôte que le temps de se nourrir : par exemple les Ceratophyllus d'oiseaux. 
son hôte. En Europe, elle n'est connue que d'Italie (Bezzi) et de France. Les références pour notre pays sont les suivantes:

- Mâles et femelles de Haute-Savoie, récoltés par Claudius Côte, « on a bear recently shot by him » (Jordan et Rothschild, 1920).

- Une femelle de Savoie, sur Meles meles, récoltée par A. Rettig en juin 1913 à St-Rémy (-de-Maurienne) (Hopkins et Rothschild, 1956).

Il est possible que le blaireau, hôte aberrant mais présentant des convergences écologiques avec l'ours, ait pu constituer un hôte vicariant, sans doute temporaire, au moment de la disparition de l'ours alpin français.

Pour ce qui concerne la première référence (captures de C. Côte), le $\mathrm{D}^{r}$ Couturier, meilleur spécialiste actuel de l'ours brun, la considère comme extrêmement douteuse (in litt., 12-12-70 et 20-12-70). Il ne pense pas que «...Claudius Côte..., qui avait le même guide de montagne que (lui)... ait jamais tué un ours, surtout en Haute-Savoie ». Toutefois, Hainard (1948) rapporte, d'après les dires de Claudius Côte lui-même, comment ce dernier a tué au début du siècle deux ours à Epierre (Savoie). Epierre est à moins de $10 \mathrm{~km}$ au nord de St-Rémy-de-Maurienne, déjà cité.

Il serait évidemment du plus haut intérêt de pouvoir examiner, en hiver, quelques ours pyrénéens. Il est vraisemblable qu'ils hébergent cette rare espèce, holarctique comme son hôte.

Une autre espèce doit être recherchée dans les Alpes françaises et peut-être les Pyrénées: il s'agit de $C$. mirabilis Ioff et Argyropoulo (1934) connue des deux rives de la Caspienne dans les régions montagneuses du Caucase et du Kopet-Dag, récemment trouvée en Suisse, dans le Valais, vers $1550 \mathrm{~m}$ (Smit, 1966). C'est un parasite du renard et de la fouine.

\section{Chaetopsylla globiceps (Taschenberg, 1880)}

Nous avons déjà noté (Beaucournu, 1968) que la mention de cette espèce en France reposait sur des erreurs bibliographiques ou, comme nous l'avons montré, de détermination; notre pays, toutefois, était certainement concerné par la distribution de cette espèce qui recouvre, en particulier, toute l'Europe, sauf les Iles britanniques et la Péninsule ibérique.

Nous venons effectivement de trouver cette puce, mais en très petit nombre dans deux prélèvements (Hauville, Eure; Combronde, Puy-de-Dôme). Ceci est étonnant: son hôte est le renard et nous en avons étudié, rappelons-le, environ 80 . Il est vraisemblable que C. globiceps n'habite qu'une partie de notre pays. Elle paraît manquer dans toute la moitié sud où nos récoltes sont relativement abondantes et en particulier dans les Alpes.

Nos 4 exemplaires ( 2 mâles, 2 femelles) furent prélevés en décembre et en avril: ils proviennent de deux renards.

Chaetopsylla homoea Rothschild, 1906

Cette puce, que nous avons signalée de France pour la première fois en 1971, est maintenant la plus abondante du genre Chaetopsylla dans nos relevés, bien qu'elle paraisse localisée à la région alpine. 
Elle n'est connue que d'Asie centrale [Kirghizie (Rothschild, 1906), Caucase, Tian-Chan, Altai, Sajan, Transbaikalie, Tibet, Mongolie (Smit, 1966)], de Suisse (Jordan et Rothschild, 1920 ) et de France (Beaucournu et Gilot, 1971). Nos captures proviennent actuellement de quatre départements: Savoie, Isère, Hautes-Alpes, Alpes de Haute-Provence.

Il semble s'agir d'une espèce exclusivement altitudinale. A notre connaissance, les deux stations les plus élevées sont Avers (Suisse), grotte d'Unter-Rotloch à $2550 \mathrm{~m}$ (Strinati et Aellen, 1964) et Valloire (Savoie) à $2300 \mathrm{~m}$ (Beaucournu et Gilot, rec.). Dans les Alpes de Haute-Provence où nous avons des prélèvements abondants en haute, moyenne et basse altitude, nous ne trouvons $C$. homoea qu'au-dessus de $1000 \mathrm{~m}$ : la commune la moins élevée est Barcelonnette à $1132 \mathrm{~m}$. Dans les stations plus basses [Digne $(600 \mathrm{~m})$, Sisteron $(480 \mathrm{~m})$ ], cette espèce est remplacée par $C$. rothschildi. Aucun de nos prélèvements n'a encore montré, à la fois, ces deux puces.

Chaetopsylla homoea est donnée par Smit (1966) comme parasitant essentiellement, en Suisse, la belette (Mustela nivalis), mais «manifestement non exceptionnelle sur le renard». Nos données sont faussées par le fait que la majeure partie des hôtes étudiés dans la région alpine furent des renards qui fournissent la quasi-totalité de nos captures, soit 716 exemplaires. Un blaireau, une fouine et une hermine étaient également parasités. Bien que l'on ne puisse guère faire état de chiffre aussi bas, une seule $M$. erminea, sur 3 capturées à la fin de septembre vers $2300 \mathrm{~m}$, était parasitée (6). Quant à la fouine, capturée en janvier vers $1750 \mathrm{~m}$, dans le Briançonnais, elle livra 30 mâles et 40 femelles ce qui implique bien qu'il s'agit-là d'un hôte normal.

Nos récoltes, au total, regroupent 796 exemplaires de cette «rare» espèce. Le sexratio est très nettement en faveur des femelles $(0,54)$.

\section{Chaetopsylla matina (Jordan, 1925)}

Cette espèce fut décrite par Jordan des Hautes-Pyrénées (Cauterêts) et est encore très mal connue: Hopkins et Rothschild (1956) ajoutent quelques nouvelles captures provenant de la même commune (7). Skuratowicz (1963) signale la récolte à Bialowieza (NordEst de la Pologne) d'1 mâle et de 6 femelles. Enfin Suciu (1967) l'identifie de Roumanie d'après 1 mâle et 9 femelles.

Nous n'avons encore obtenu cette espèce qu'une fois en provenance du Massif du Carlitt, dans la région du lac de la Bouillouse (Pyrénées-Orientales): la récolte comportait 1 mâle et 2 femelles prélevés en janvier entre 1300 et $1800 \mathrm{~m}$ d'altitude.

Les hôtes signalés par Jordan, puis par Hopkins et Rothschild, sont Martes martes et $M$. foina; les exemplaires polonais et roumains proviennent également de $M$. martes. Avec nos exemplaires, apparaît un nouvel hôte, Vulpes vulpes. De nouvelles captures sont nécessaires pour savoir s'il s'agit, ou non, d'un hôte accidentel (8).

Le sex-ratio de cette espèce est, si l'on en juge par les données de la littérature, nettement en faveur des femelles.

(6) Deux belettes capturées en décembre et août vers $1800 \mathrm{~m}$, respectivement dans la région de Briançon et le Queyras, n'hébergeaient aucun parasite spécifique.

(7) «Pont d'Espagne» signalé comme commune autonome par ces auteurs est sur le territoire de Cauterets.

(8) Hopkins et Rothschild (1956) signalent des captures, également faites par J. Mousquès, sur hérisson et taupe. Il s'agit certainement d'erreurs, ou de contaminations, de prélèvements. 
Chaetopsylla rothschildi Kohaut, 1903

La répartition de Chaetopsylla rothschildi semble essentiellement couvrir la bordure européenne et proche-orientale de la méditerranée. D’Ouest en Est, elle est connue de France, Yougoslavie, Tchécoslovaquie, Hongrie, Roumanie, Turquie, Liban et Caucase.

En France, elle est signalée de l'Allier (Rothschild, 1911) et de Haute-Garonne (Beaucournu, 1968). Nous l'avons retrouvée dans ce département et la signalons également de Dordogne, du Lot-et-Garonne, du Tarn et des Alpes de Haute-Provence. Toutes les captures ont été faites à basse altitude, de décembre à mars.

La fouine est manifestement son principal hôte puisqu'environ 25 sur 35 de ces mustélidés en étaient porteurs avec un total de 183 puces. Une douzaine de renards étaient également parasités totalisant 62 specimens. Un mâle de $C$. rothschildi a, enfin, été récolté sur une genette, Genetta genetta, la seule étudiée. C'est, à notre connaissance, la première mention d'un siphonaptère sur la genette, en Europe.

Nos 246 exemplaires se répartissent en 126 mâles et 120 femelles, soit, sensiblement, un sex-ratio équilibré.

\section{Chaetopsylla trichosa Kohaut, 1903}

C. trichosa est l'espèce du genre la plus répandue en Europe. Connue de pratiquement toute la zone paléarctique, c'est la seule Chaetopsylla signalée des Iles britanniques et d'Espagne.

Nos captures, bien qu'encore clairsemées, montrent que $C$. trichosa est présente dans toute la France continentale. En dehors des départements déjà connus (Haute-Pyrénées : Hopkins et Rothschild, 1956; Mayenne: Beaucournu et Matile, 1963), nous la signalons du Haut-Rhin, Yonne, Isère, Hautes-Alpes, Alpes de Haute-Provence, Haute-Garonne, Tarnet-Garonne, Tarn et Lot-et-Garonne.

En altitude, nous ne l'avons encore notée que des Alpes où elle est très nettement dominée numériquement, par $C$. homoea. Dans la région de Briançon, par exemple, le rapport est de 1 pour 45. En plaine, ce rapport n'est guère plus élevé: dans le Lot-etGaronne, nous en trouvons environ 1 pour $20 \mathrm{C}$. rothschildi. Il faut souligner que ce sont des chiffres globaux regroupant l'ensemble des captures. Or, C. trichosa est connue comme parasitant essentiellement le blaireau et cet animal est en relative minorité dans nos récoltes. Huit blaireaux étaient parasités par $C$. trichosa et ont livré 91 spécimens. Deux autres carnivores nous ont fourni cette puce: le renard et la fouine: 17 renards ont donné 49 exemplaires; 2 fouines en hébergeaient chacune un. Ces chiffres confirment bien que le blaireau est l'hôte principal de $C$. trichosa.

$\mathrm{Au}$ total nous avons récolté ou identifié 142 exemplaires de cette puce: 71 mâles et 71 femelles (S.R. = 1).

\section{Ceratophyllidae}

Ceratophyllus lunatus lunatus Jordan et Rothschild, 1920

Cette espèce, seul Ceratophyllus (sensu lato) parasite de mammifères, est inféodée à l'hermine Mustela erminea. 
La répartition est arctique et subarctique dans toute la zone holarctique : le Groenland et l'Amérique du Nord sont habités par C.l. tundrensis Holland, 1948 ; l'Europe et le Nord de l'U.R.S.S. par la forme nominale à répartition typiquement boréo-alpine. Sous nos latitudes, elle n'est connue que d'une station en Suisse (Zermatt, Valais, $1650 \mathrm{~m}$ : J. et R., 1920 ) et de trois en France: Bonneval-sur-Arc (Savoie) (9) (Jordan et Rothschild, 1922), Valloire (Savoie), Granges du Galibier, $2300 \mathrm{~m}$ (Beaucournu et Gilot, rec.), Allos (Alpes de Haute-Provence), Cirque du Laus, $2100 \mathrm{~m}$ (Beaucournu et Gilot, 1971).

L'hôte des exemplaires suisses est incertain (cf. Smit, 1966): on sait seulement qu'il s'agit d'un petit mustélidé, belette ou hermine. Tous les spécimens français proviennent de l'hermine qui est, d'ailleurs, plus abondante dans les Alpes en altitude, que la belette. Parmi nos captures provenant de cette région, 3 hermines sur 5 étaient parasitées 57 autres, par contre, de diverses régions de France, dont 3 venant de haute altitude dans les Pyrénées, n'étaient pas parasitées. De même, aucune des 52 belettes étudiées, dont 3 provenant des Alpes, n'a livré cette puce.

Le cycle annuel de $C$. lunatus n'est pas connu. Les exemplaires suisses (3 mâles) furent capturés en décembre; ceux de France en juillet ( 3 femelles), en septembre (1 mâle, 3 femelles) et en novembre («a pair», Jordan et Rothschild, 1922).

Il serait intéressant de pouvoir étudier quelques litières d'hermines: C. lunatus est-il comme les Ceratophyllus d'oiseaux, une puce de nid ou, comme les genres voisins inféodés aux mammifères, une espèce sans préférence écologique très précise sous cet angle et pratiquement aussi abondante dans les litières que dans la fourrure de ses hôtes? Nous penchons vers cette éventualité.

\section{Paraceras melis melis (Walker, 1856)}

Paraceras melis est une espèce paléarctique largement répandue de l'ouest européen à la Chine. Cette puce se rencontre dans toute la France.

Le manque d'homogénéité de nos récoltes rend difficile une schématisation. On peut néanmoins relever que nous avons des captures de cette espèce durant tout le cycle annuel avec présence de femelles ovigères et d'individus néonates aussi bien en été qu'en hiver. Lewis (1964) au Liban note, à l'inverse, qu'il s'agit d'une puce exclusivement hivernale, tous ses exemplaires (140) ayant été récoltés en janvier principalement, février et mars. Paraceras $m$. melis, précise cet auteur, est la puce dominante sur le blaireau pendant une période limitée des mois les plus froids, mais elle est remplacée par Pulex irritans durant la plus grande partie de l'année.

Paraceras melis n'est pas, apparemment, une espèce d'altitude. Nous ne possédons que 6 exemplaires (sur 442) capturés entre 1000 et $1500 \mathrm{~m}$ d'altitude. Le blaireau, toutefois, principal hôte de cette puce, n'est pas abondant au-dessus de $1000 \mathrm{~m}$.

$P$. melis est indiscutablement inféodé à ce carnivore : 271 exemplaires proviennent de 30 blaireaux sur 35 examinés. Il n'est pas exceptionnel cependant sur d'autres carnivores terrestres ou rupicoles, de taille moyenne: il s'agit sans doute de vicariance écologique. En premier lieu vient le renard sur qui il est fréquemment trouvé mais en petit nombre (71 sur une vingtaine de renard parasités). Les autres carnivores figurant dans nos récoltes sont la fouine ( 2 fois avec 2 exemplaires en tout) et le putois (un seul spécimen).

Paraceras melis est, comme Pulex irritans, fréquemment récolté sous terre et dans les mêmes conditions: c'est souvent dans les plis de la combinaison de spéléologue qu'on

(9) L'altitude de cette commune est $1.870 \mathrm{~m}$. 
la récolte, ce qui n'est jamais le cas pour d'autres puces peu rares dans les cavités, Spilopsyllus cuniculi (Dale) par exemple: ceci semble indiquer que Paraceras pourrait piquer l'homme.

Si $P$. melis est souvent trouvé sur le sol nu, il est également fréquent dans les litières souterraines (10) de Meles meles. Ceci montre qu'il ne s'agit pas d'une puce de fourrure, comme par exemple les Chaetopsylla chez les carnivores ou les Paleopsylla chez les insectivores.

Nos récoltes ont porté sur 442 exemplaires montrant un sex-ratio nettement en défaveur des mâles (177 mâles, 265 femelles) : S.R. $=0,66$. D'après les observations de Lewis, au Liban, il semblerait que les mâles apparaissent les premiers. Nos observations ne nous permettent encore ni d'infirmer ni de confirmer ce fait pour la France.

\section{Monopsyllus sciurorum sciurorum (Schrank, 1803)}

Bien que $M$. sciurorum soit essentiellement un parasite de rongeurs «arboricoles », nous suivons ici l'opinion de Smit (1966) qui considère les espèces du genre Martes comme hôtes secondaires de cette puce.

L'écureuil, Sciurus vulgaris, principal représentant européen de ce groupe de rongeurs, sera régulièrement et abondamment parasité. Les gliridés, lorsqu'ils habiteront les arbres (Muscardinus, Glis et tout particulièrement Eliomys), seront également de très bons hôtes. Dans tout l'ouest atlantique français, $M$. sciuronum est la puce classique du lérot, Eliomys quercinus. Cet animal changera de faune dans deux circonstances: dans les zones d'influence méditerranéenne où Myoxopsylla laverani supplante Monopsyllus, et en haute altitude où le lérot est exclusivement un habitant des fentes de rocher (ce qui constitue son habitat primitif). Il hébergera alors, outre Myoxopsylla, de nombreuses puces, à spécificité écologique, de rongeurs liés à ce biotope, en particulier du campagnol Microtus nivalis. On peut superposer, par contre, les aires de répartition de l'écureuil et de Monopsyllus sciurorum. En France, une autre puce est également inféodée à ce dernier: Tarsopsylla o. octodecimdentata (Kolenati, 1863). Beaucoup plus rare, cette espèce, chez nous, semble limitée aux zones d'altitude.

La fréquence de Monopsyllus sciurorum sur un prédateur sera fonction du mode de vie de celui-ci. Il va être assez difficile de faire le partage entre parasites déserteurs d'une proie et, vicariants «égarés» sur un prédateur ayant un nid aérien, ce nid pouvant même être emprunté à un rongeur arboricole.

Il faut souligner que Monopsyllus est essentiellement une puce de nid. Celui d'un écureuil en abrite couramment plusieurs dizaines, voire plusieurs centaines: nous avons récolté un ancien nid de ramier (Columba palumbus), réoccupé par un lérot, recouvert d'une couche frémissante et uniforme de cette puce.

Par ordre de fréquence nous trouvons comme carnivores parasités: 1 fois une belette ( 1 puce), 1 fois une hermine ( 2 puces), 2 fois un putois (nombreuses puces: nous en parlerons plus loin), 8 fois une marte ( 59 puces) et 12 fois une fouine ( 39 puces). On peut souligner que, en dehors de la saison d'apparition des Chaetopsylla, Monopsyllus est la puce la plus souvent notée sur la fouine, mais, d'après les quelques relevés que nous venons de donner, la marte semble un meilleur hôte que celle-ci, son écologie d'animal arboricole l'expliquant en partie.

(10) Nous avons eu l'occasion d'étudier une litière de blaireau placée à l'extérieur des galeries, à l'air libre. Elle ne renfermait que des Ixodidés. 
Le cas d'un des putois est très particulier. Il s'agissait d'un subadulte trouvé mort le long d'une falaise karstique sans lésion externe ou interne décelable, la mort remontant à quelques heures seulement, porteur d'une soixantaine d'imagos, de plus de cent larves à divers stades et d'une nymphe fixée aux poils. Le parasitisme de l'hôte par les larves est exceptionnel, hormis le cas des animaux en hibernation et celui d'Hoplopsyllus glacialis, parasite du lièvre arctique, dont les larves, d'après Freeman et Madsen (1949), vivraient normalement sur cet animal en compagnie des imagos. A l'état naturel, nous ne connaissons dans nos régions aucun autre cas que celui que nous venons de rapporter (11).

Monopsyllus sciurorum est abondant tout au long de l'année en-dessous de $800 \mathrm{~m}$. Nous n'avons pas de données suffisantes en altitude pour savoir ce qu'il en est en hiver.

Calculée sur plus de 1600 exemplaires collectés sur Sciurus, Eliomys, Glis ou dans leurs nids, le sex-ratio est de 0,72 (0,69 pour les puces de nids; 0,94 pour celles récoltées sur les hôtes). Sur les prédateurs, celui-ci, pour 156 puces prélevées dans la fourrure, tombe à 0,32 . Cette variation apparente du sex-ratio en fonction des hôtes, spécifiques ou non, n'a pas, à notre connaissance, été étudiée (12).

\section{Puces diverses non spécifiques}

Vingt-deux autres espèces de Siphonaptères ont été récoltées plus ou moins fréquemment sur des carnivores. Deux Pulicidae parasites de proies moyennes (lapin et hérisson) vont se retrouver surtout sur les prédateurs les plus grands, renard en particulier. Les parasites de micromammifères vont, presque uniquement, être récoltés sur les très petits carnivores, belette et hermine.

Nous avons regroupé ces données dans le tableau 2.

\section{II. - LES HOTES.}

Vu sous l'angle du prédateur, il nous paraît intéressant de voir comment se répartissent ces parasites sur les divers prédateurs étudiés, en fonction de leur spécificité et de leur écologie.

Nous y avons inclu le chien et le chat domestique en raison de leur intérêt épidémiologique, bien que nos relevés n'aient pas été systématiques.

(11) D'assez nombreux cas, par contre, sont connus concernant des animaux domestiques ou captifs. Brumpt (1910) rapporte le cas d'un furet (Mustela putorius furo) présentant le même type de parasitisme par Ceratophyllus sp. (= Nosopsyllus fasciatus J.-C. B. det.), mais il s'agissait là d'une bête malade. Divers auteurs (Austin, Leuckart, Neumann in Brumpt op. cit.; Galli-Valerio, 1931) signalent des faits identiques sur des chiens et chats domestiques. Le Laboratoire d'Entomologie du Muséum National d'Histoire Naturelle possède également de nombreuses larves et œufs de Ctenocephalides $s p$. prélevés sur chien. Il est presque toujours noté que ces animaux étaient cachectiques ou malades. Railliet et Henry (1910) rapportent un cas analogue, chez une femme, dû cette fois à Pulex irritans: nous n'avons pu lire cette observation citée, sans autre référence, par Brumpt (1949).

(12) Le cas d'Archaeopsylla erinacei (Bouché, 1835) est peut-être analogue mais encore basé sur des chiffres trop faibles. Calculé sur environ 1500 spécimens, le S.R. des puces prélevées sur l'hôte spécifique (le hérisson) est de 0,7 ; nous avons 11 exemplaires récoltés sur prédateurs (renard, essentiellement) groupant 10 femelles et 1 seul mâle. 


\section{Tableau II}

PUCES DIVERSES NON SPÉCIFIQUES

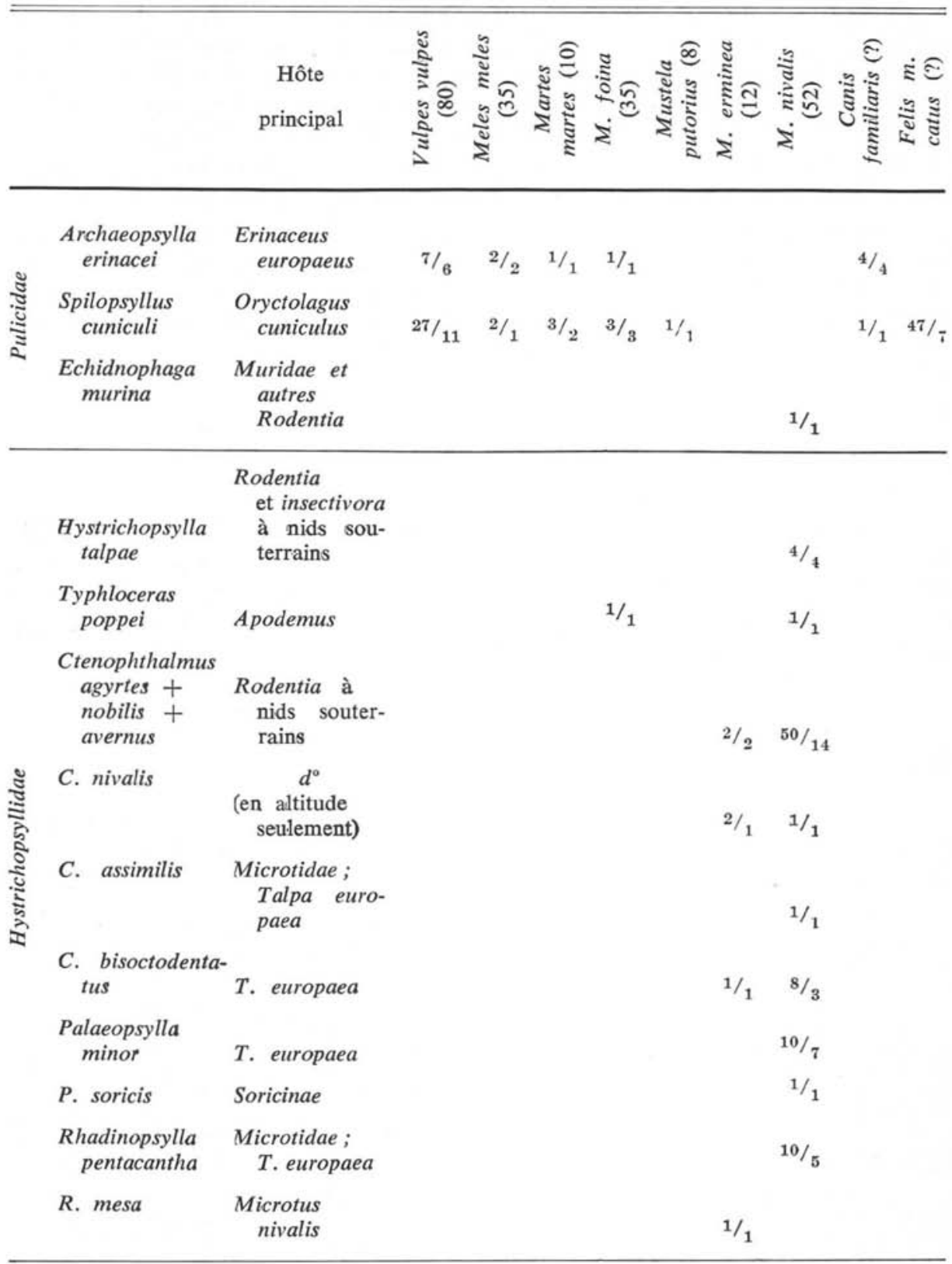


Tableau II (suite)

PUCES DIVERSES NON SPÉCIFIQUES

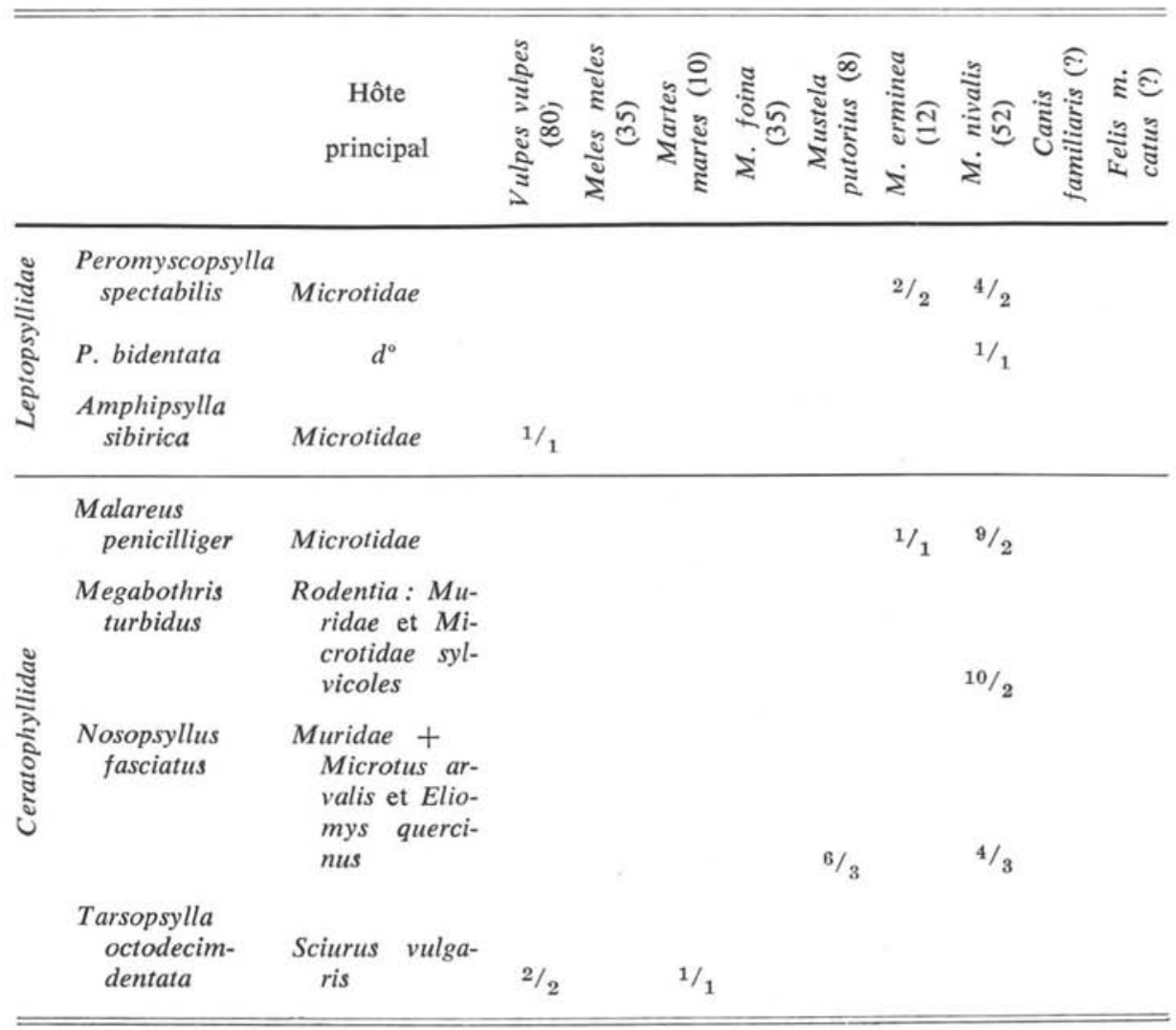

N. B.: Les puces récoltées dans les litières ne sont pas intégrées dans ce tableau.

Le chiffre supérieur indique le nombre de parasites récoltés, l'inférieur le nombre d'hôtes parasités.

\section{Canidae}

\section{Vulpes vulpes}

Le renard présente une riche faune de puces spécifiques. En Europe occidentale, c'est l'hôte principal de Chaetopsylla globiceps et peut-être aussi de $C$. mirabilis, cette dernière espèce manquant encore à notre faune. C. homoea, considérée comme parasitant surtout les mustélidés, est très abondante sur cet animal qui représente certainement l'un de ses hôtes principaux, en France. 
Deux autres Chaetopsylla, bien que fréquentes, ne parasitent pas électivement le renard: ce sont $C$. trichosa et $C$. rothschildi. Nous signalons enfin pour la première fois la rare $C$. matina sur cet hôte.

Pulex irritans et Ctenocephalides canis vont représenter l'élément le plus important, épidémiologiquement, des puces de $V$. vulpes, de par les échanges d'ectoparasites qui vont pouvoir s'effectuer entre renards, autres carnivores aussi bien domestiques que sauvages, et homme. Ces deux puces, présentes toute l'année, sont d'excellents vecteurs de diverses maladies bactériennes et parasitaires: nous avons signalé en certaines régions (Sud-Ouest de la France) la fréquence des $P$. irritans hébergeant des larves infectantes de Dipetalonema reconditum.

Paraceras melis, enfin, bien que non spécifique du renard n'est pas rare sur cet animal.

Les déserteurs comportent d'abord, par ordre de fréquence, deux pulicidés, Archaeopsylla erinacei et Spilopsyllus cuniculi : les hôtes normaux (hérisson et lapin) figurent dans les proies classiquement admises du renard et il y a en outre, dans les deux cas, possibilité de contamination écologique. Deux spécimens de Tarsopsylla, parasites de l'écureuil, et un de puce de microtidé (Amphipsylla sibirica ? sepifera Jordan et Rothschild, 1920) complètent la faune observée sur le renard.

Il est indiscutable que les hôtes normaux de ce maigre inventaire de déserteurs no représentent pas le régime de ce carnivore: les micromammifères constituent une part très importante de son alimentation (Corbet, 1966; Vericad, 1970). Les pulicidés inféodés au hérisson et au lapin, s'ils ne s'adaptent pas au renard, du moins survivent-ils un certain temps sur cet hôte ; les puces de micromammifères, elles, ne s'y égarent même pas. Il s'agit à la fois de facteurs écologiques et morphologiques: ni le microclimat de la litière, ni l'écoumène représenté par la fourrure du renard ne devant leur convenir.

\section{Canis familiaris}

Nos relevés, trop peu abondants, montrent essentiellement deux faits intéressants:

- la fréquence de Pulex irritans sur le chien;

- la différence de faune entre le chien de «salon» et celui menant une vie moins dépendante de l'homme: le premier est surtout parasité par la puce euryxène et synanthrope Ctenocephalides felis, le second par $C$. canis, sténoxène de canidé.

\section{Viverridae}

\section{Genetta genetta}

L'unique exemplaire étudié à ce jour confirme, par le parasite rencontré Chaetopsylla rothschildi, les convergences écologiques existant entre la genette et la fouine.

De plus amples données seraient utiles mais seront difficiles à obtenir puisque, à juste titre, la genette est protégée. 


\section{Felidae}

\section{Felis maniculata catus (= domestica)}

Bien que vivant en milieu artificiel où 3 puces de carnivores se côtoient plus ou moins, le chat n'est parasité que par Ctenocephalides felis.

Parmi les déserteurs, nous n'avons noté, mais avec fréquence chez les chats vivant à la campagne, que Spilopsyllus cuniculi. Il y a là encore discordance entre le régime et les déserteurs observés, de nombreux petits rongeurs entrant dans la liste de ses proies. Les passereaux, classiquement chassés, n'entrent pas en compte puisqu'ils ne sont parasités que par des puces de «nid».

\section{Mustelidae}

\section{Meles meles}

Trois siphonaptères constituent la faune normale et spécifique du blaireau : Chaetopsylla trichosa, Paraceras melis et Pulex irritans.

Les déserteurs sont exceptionnels, cette rareté étant peut-être en relation à la fois avec la fourrure du blaireau composée de jarres très épais et son régime omnivore riche en végétaux et invertébrés (Hainard, 1948 ; Corbet, 1966; Vericad, 1970).

\section{Martes martes}

Aucun parasite spécifique (Vermipsyllidae en particulier) n'a été récolté sur les martes que nous avons étudiées. Nous n'avons noté que des déserteurs: Archaeopsylla erinacei, Spilopsyllus cuniculi, Monopsyllus sciurorum et Tarsopsylla octodecimdentata.

Smit (1966), nous l'avons dit, considère martes et fouines comme hôtes secondaires de Monopsyllus sciurorum, qui fut d'ailleurs la seule puce abondante sur Martes martes dans nos récoltes.

On doit cependant noter que des Chaetopsylla sont connues de la marte: C. matina surtout, et plus accessoirement $C$. rothschildi.

\section{Martes foina}

Cette espèce, nettement plus fréquente que la précédente, nous a livré abondamment une puce spécifique, Chaetopsylla rothschildi. D'après la bibliographie, elle est également l'hôte de $C$. mirabilis et de $C$. matina.

Dans nos récoltes, 6 autres puces de carnivores figurent, en petit nombre, sur cet animal: Pulex irritans (3 fois), Ctenocephalides felis ( 2 fois), C. canis (2 fois), Paraceras melis ( 2 fois) Chaetopsylla trichosa (2 fois) et $C$. homoea ( 1 fois).

Le nid, aérien mais bien protégé (tronc d'arbre, fissure de falaise, vieux bâtiments), le régime carnivore éclectique et sans doute aussi la texture fine de sa fourrure vont permettre la récolte sur cet animal de divers déserteurs: Archaeopsylla erinacei, Spilopsyllus cuniculi (ces deux espèces étant des contaminations de prédation), mais surtout Monopsyllus sciurorum dont nous avons déjà parlé à propos de la marte. 


\section{Mustela putorius}

Cet animal banal et très répandu en plaine ne figure cependant que par 8 captures dans nos relevés.

Bien peu de références attribuent au putois une puce pouvant passer pour spécifique: Callopsylla (Paracallopsylla) kaznakovi (Wagner, 1929), Chaetopsylla rothschildi et $C$. hornoea sont citées d'U.R.S.S. : pour autant qu'on puisse le savoir, il s'agissait d'ailleurs du putois Mustela eversmanni et non de Mustela putorius, seule espèce des deux présente en France.

Les siphonaptères que nous avons récoltés sont manifestement des déserteurs (Spilopsyllus cuniculi) ou des parasites de carnivores (Paraceras, Ctenocephalides) mais en trop petit nombre pour que cela soit significatif. La seule espèce qui se soit comportée comme une puce spécifique est Monopsyllus sciurorum, rencontrée 2 fois sur 8 hôtes examinés, dont une fois en grande abondance. Cela est d'autant plus notable que Mustela putorius n'a pas une écologie pouvant justifier une telle contamination : en particulier ce n'est pas un grimpeur. Smit (1969), qui, avec de nombreux auteurs, signale également cette association hôte-parasite, rapporte la contamination de visons d'élevage (Mustela vison) « in immense numbers and causing much annoyance to their hosts... .

\section{Mustela nivalis}

La belette est l'un des carnivores les mieux représentés dans nos captures et nous en avons récoltés dans presque toute la France et à toutes les altitudes.

Jusqu'à maintenant aucune puce spécifique n'a été récoltée. Nos captures ne comportent que des déserteurs de rongeurs souterrains, surtout de microtidés, et d'insectivores, taupes essentiellement: ceci confirme le régime et l'écologie de la belette, véritable commensal des galeries de Talpa, Arvicola, Microtus, etc... L'incidence de ce petit carnivore dans l'épidémiologie de l'arbovirose E.E.C. dont le réservoir animal serait la taupe (Taytsch et Wroblewska, 1958; Grulich, 1962 ; Kozuch, Grulich et Nosek, 1966...) peut donc être particulièrement importante. Rappelons que cet arbovirus fut isolé de puces de petits mammifères, en Pologne, par Przesmycki et coll. (1956).

Il est vraisemblable qu'un certain nombre des espèces notées peuvent se reproduire aux dépens de la belette. Nous en avons eu la preuve avec Nosopsyllus fasciatus. Nous avons pu étudier une belette et son nid, l'un comme l'autre nous livrant cette espèce: 160 puces furent extraites de la litière parmi lesquelles $159 \mathrm{~N}$. fasciatus. D'autre part, la fréquence relative de Ctenophthalmus, Megabothris et même de Rhadinopsylla pentacantha (Rothschild, 1897), cependant peu abondante sur ses hôtes normaux, les microtidés, nous font penser que la belette peut constituer pour ces parasites un vicariant écologique.

\section{Mustela erminea}

L'hermine est répandue partout, mais, sauf en altitude, sa densité est faible. Ce n'est qu'en zone montagneuse, d'ailleurs, qu'elle livre des parasites spécifiques: Ceratophyllus l. lunatus et Chaetopsylla homoea, et ceci seulement dans les Alpes, jusqu'à présent.

Les autres puces notées sont des déserteurs de rongeurs, essentiellement de microtidés: Ctenophthalmus et Malareus en particulier. 


\section{Bibliographie}

Bain (O.) et Beaucournu (J.-C.), 1973. - Larves infestantes de Dipetalonema reconditum chez Pulex irritans, récoltés sur des renards dans le sud-ouest de la France. Ann. Parasit. hum. comp., 48 (à paraître).

Beaucournu (J.-C.), 1968. - Catalogue provisoire des siphonaptères de la faune française. Ann. Soc. Entom. France, n. s., 4, 615.

- et GrLot (B.), 1971. - Additions au Catalogue provisoire des siphonaptères de la faune française. Bull. Soc. entom. France, 76, 46.

— et Matile (L.), 1963. - Contribution à l'inventaire faunistique des cavités souterraines de l'ouest de la France. III: liste des espèces; bibliographie. Ann. Spéléologie, 18, 519.

— et Rault (B.), 1962. - Contribution à l'étude des siphonaptères de mammifères dans la moitié orientaie des Pyrénées. Vie et milieu, 13, 571.

Brumpt (E.), 1910. - Précis de Parasitologie, Masson et $C^{\text {te }}$ édit., Paris.

—, 1949. - Précis de Parasitologie, 2 vol., Masson et $C^{\text {ie }}$ édit., Paris.

CoRBet (G. B.), 1966. - The terrestrial mammals of western Europe, Foulis et $\mathrm{C}^{\text {te }}$ éd., London.

Freeman (R. B.) et Madsen (H.), 1949. - A parasitic flea larva. Nature, 164, 187.

Galli-Valerio (B.), 1931. - Notes de Parasitologie. Zbl. Bakt., 120, 98.

Grulich (I.), 1962. - On the european mole (Talpa europaea L.) in focuses of tick encephalitis in Czechoslovakia. Symposium theriologicum, Brno, Nakl. ČSAV, Praha, 141.

HaINARD (R.), 1948. - Les mammifères sauvages d'Europe. I: Insectivores, Cheiroptères, Carnivores, Delachaux et Niestlé éd., Neuchâtel et Paris.

Hopkins (G. H. E.) et Rothschild (M.), 1956. - An illustrated catalogue of the Rothschild collection of fleas. II : Vermipsyllidae to Xiphiopsyllidae. Trust. British Museum (Nat. Hist.), London.

Jordan (K.) et Rothschild (N. C.), 1920. - A preliminary catalogue of the Siphonaptera of Switzerland. Ectoparasites, 1, 78.

— et -, 1922. - New siphonaptera. Ectoparasites, [1, 266.

Kozuch (O.), Grulich (I.) et NoseK (J.), 1966. - Experimental infection of the mole with tick borne encephalitis virus. Jl. Hyg. Epidemiol. Microbiol. Immunology, 10,120 .

Lewis (R. E.), 1964. - Additional records of fleas from Lebanon, Syria and Jordan. Proc. R. ent. Soc. Lond. (A.), 39, 157.

Przesmycki (F.), Taytsh (Z.), Wroblewska (Z.) et Lachmajer (J.), 1958. - Study of a natural focus of tick-borne encephalitis in the Puszcza Bialowieska National Park. W.H.O., 180 [in Lachmajer (J.), 1959, Bull. Inst. Mar. Med. Gdansk, $10,5]$.

Rioux (J.-A.) et Golvan (Y.J.), 1969. - Epidémiologie des leishmanioses dans le sud de la France. I.N.S.E.R.M., Monographie $n^{\circ} 37$, Paris.

Rothschild (N. C.), 1906. - Some new exotic fleas. Entom. Monthly mag., 17, 221. 
-, 1911. - Liste des siphonaptèra du Muséum d'Histoire Naturelle de Paris accompagnée de descriptions des espèces nouvelles. Ann. Sc. nat. (Zool.), 12, 203.

SKURATowicz (W.), 1963. - Chaetopsylla matina (Jordan), a species of Aphaniptera new to the polish fauna. Bull. Acad. polonaise Sc., 11, 527.

Smit (F. G. A. M.), 1960. - Records of Siphonaptera from the Pyrénées, with the description of a new species. Proc. $R$. ent. soc. London, 29, 11.

-, 1966. - Insecta helvetica. I: Siphonaptera, Lausanne.

-, 1969. - A catalogue of the Siphonaptera of Finland with distribution maps of all fennoscandian species. Ann. Zool. Fenn., 6, 47.

Strinati (P.) et Aellen (V.), 1964. - Faune actuelle de deux grottes suisses de haute altitude. Rass. Speleol. ital., 19, 1.

SuciU (M.), 1967. - Contributiuni la studiul siphonapterelor din Republica socialista romania (Siphonaptera Latreille, 1798). Soc. St. nat. geogr. R.S.R., comm. zoologie, 5,75 .

TAytsch (Z.) et Wroblewska (Z.), 1958. - In : Kozuch et coll., 1966.

Vericad (J. R.), 1970. - Estudio faunistico y biologico de los mamiferos del Pirineo. Publ. Centro pirenaico biol. exp., Jaca, 4. 